\title{
The High frequency induction electric iron Design of adjustable and constant temperature
}

\author{
Qiang Song,Jianguo Song,Pengtao Mu \\ Beijing University of Technology,China \\ 1015851610@qq.com
}

\begin{abstract}
Keywords:Double tube positive excitation convert; UC3845; Induction heating
\end{abstract}
\begin{abstract}
The method which adopts the XMC1300 of infineon as the master control chip, using armored $\mathrm{K}$ type thermocouple temperature acquisition, after XMC1300 A/D conversion of the improved PID algorithm control source system of constant temperature;To improve the traditional low frequency resistance wire of electric heating way, using the high frequency induction eddy current heating mode to improve the efficiency and speed of heating, without preheating time save energy; To meet the output voltage and electric power system load regulation and the requirement of fast dynamic response.Soldering iron instantaneous heating works reliably and has great effects.
\end{abstract}

\section{Introduction}

The electric iron is welding circuit to the tools, but the traditional electric iron has many problems:Low frequency resistance wire heating, long preheating time, large energy consumption, low utilization rate, can not meet the requirements of production applications.In this paper, the frequency of the operation is changed by AC-DC-AC, and the mode of heating is eddy current;Using 32 bit XMC1300 as the master chip, PID algorithm control temperature,realize constant temperature adjustable.Regulation of the power supply system is an important part of the electric iron, it is shouldering the electric iron and other systems to provide the important task of the payload. The electric iron is equivalent to the inductive load, the output voltage of the power supply, load adjustment rate, high dynamic response system.

The power supply for the two transistor forward topological structure and the design of the feedback loop power supply system in current mode PWM controller based on UC3845[1].Feedback network is composed of adjustable precision shunt regulator TL431 and linear opt coupler PC817[2].Forward the design of switching power supply has the advantages of simple structure, fast dynamic response, high load regulation rate, strong stability. Single inverter voltage waveform accurately realizes the voltage resonant inverter. The simulation and experimental results prove the rationality of the design, the stable electric iron, temperature regulation, fast response, and reliable operation.

\section{Principle and structure}

\section{Principle of Induction Heating Power Supply}

Induction heating power supply is based on the principle of electromagnetic induction, heating the work piece in the alternating magnetic field in the use of eddy current. The current through the coil produces an alternating magnetic field when the magnetic field lines within the metal work piece to be heated, the alternating magnetic field lines penetrate the metal work piece to form a loop, the induced current is in the section which can make the work piece to be local instantaneous heat for the electric iron heating purposes [3]. 


\section{The structure diagram of induction heating electric iron}

The main structure is divided into: power supply, inverter, CPU, temperature acquisition, temperature control which is shown in fig.1:

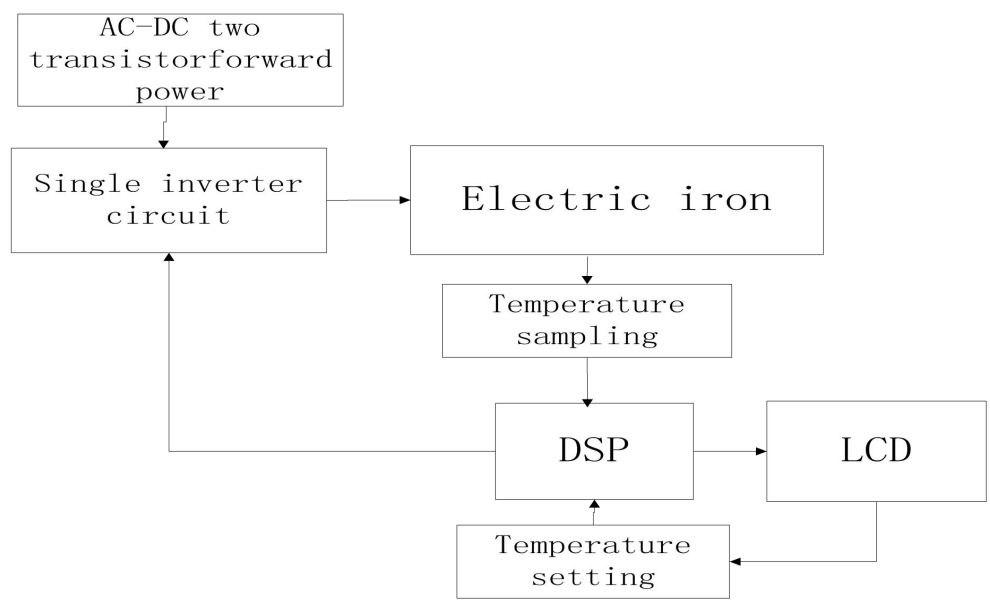

Fig. 1. Schematic Structure of Induction Heating

The main work of this paper is to design the structure of each part: the conversion of AC220VDC70V/15V dual switch forward power supply; inverter part of the realization of parallel resonance voltage to $\mathrm{AC}$ inverter, voltage resonance using eddy current of electric heater.

\section{The design of induction heating electric soldering iron hardware system}

\section{Design of two transistor forward converter power supply}

The two transistor forward converter can reduce the voltage stress of switches, two transistor forward converter as shown in figure 2. Two transistor forward converter transformer magnetic reset circuit does not need additional resetting circuits. With respect to the half bridge converter and full bridge converter, each bridge arm two transistor forward converters composed of a switch and a diode in series, there is no shoot through phenomenon to get high reliability. With the switch in series diode voltage clamp switches in the input voltage, and circuit for the excitation current of transformer, the feedback to the input power supply. In view of the above advantages, two transistor forward converter circuit topology is widely used in industry [4].

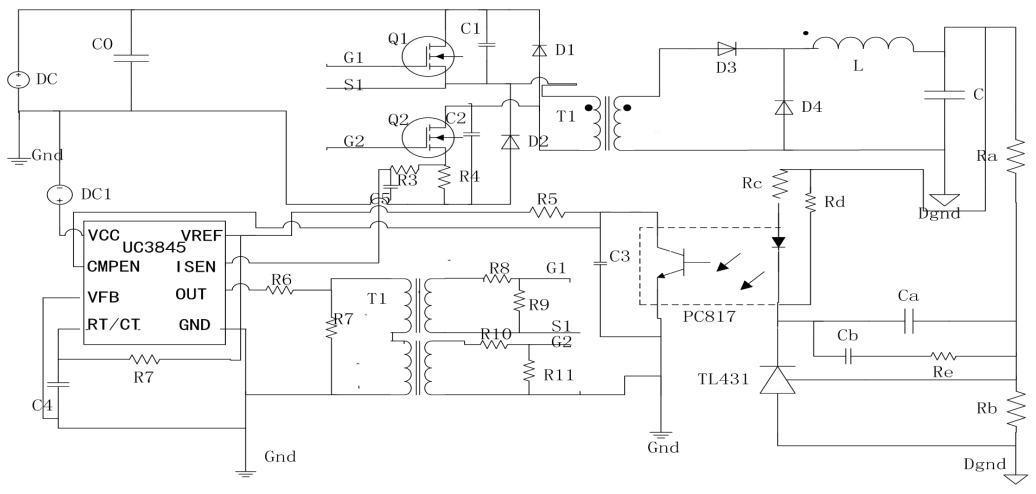

Fig. 2. Schematic Diagram of Two Transistor forward

Two transistor forward converter can be applied to high voltage, large power output. Each switch tube under maximum voltage is VI. A single forward converter, voltage stress is reduced by half. The diode D3, D4 can achieve self-reset, does not need the extra magnetic reset circuit transformer. The main effect is achieved by the DC output $220 \mathrm{~V} \mathrm{AC}$ and output $35 \mathrm{~V}$. 
The feedback network: feedback network is composed of adjustable precision shunt regulator TL431 and linear optocouplerPC817. The current PWM control chip UC3845 has some advantages like fast response speed, strong stability [5].

From Figure 2 you can see that: sampling the output voltage through TL431 comparison, PI regulation, control the size of the current through the PC817, by UC3845 to control the PWM duty cycle relation, control the Mosfet turn-on and turn off time, and then controlling the size of the output voltage through continuous adjustment of the formation of a feedback network, allowing the output the voltage stable in the expected voltage value. Two transistor forward converter make the voltage feedback adjustment speed fast, stable work.

\section{Single inverter circuit design}

The inverter part adopts the simple single inverter circuit and the load to constitute a parallel resonant circuit. Therefore, the voltage inverter for $\mathrm{AC}$ voltage generates an alternating current for the eddy current to the heater, the circuit structure as shown in fig. 3 :

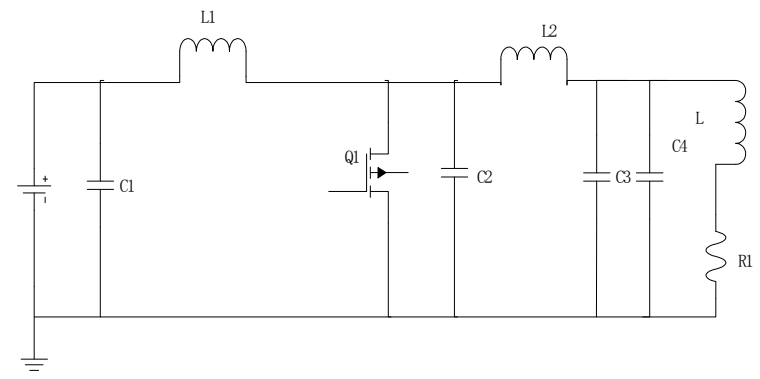

Fig. 3. Schematic Diagram Of The Inverter Circuit

When the load circuit work in the resonant state, the output current of the load induction is not large. But the current coil $\mathrm{L}$ and a capacitor $\mathrm{C}$ is great, which is $\mathrm{N}$ times as the input power supply, power supply can be realized by it. The output current waveform of the Parallel resonant inverter is AC rectangular wave. The harmonic in the load circuit generates pressure drop very small, so the load voltage waveform is close to sinusoidal wave in order to achieve the parallel inverter.

\section{Electric soldering iron temperature control system}

\section{Temperature control system block diagram}

Keeping the electric iron temperature control adopts XMC1300 as the main control chip and realtime temperature into the MCU through the analysis and comparison of the AD conversion, also using PID control algorithm to achieve constant temperature of the inverter system, the block diagram is as follows:

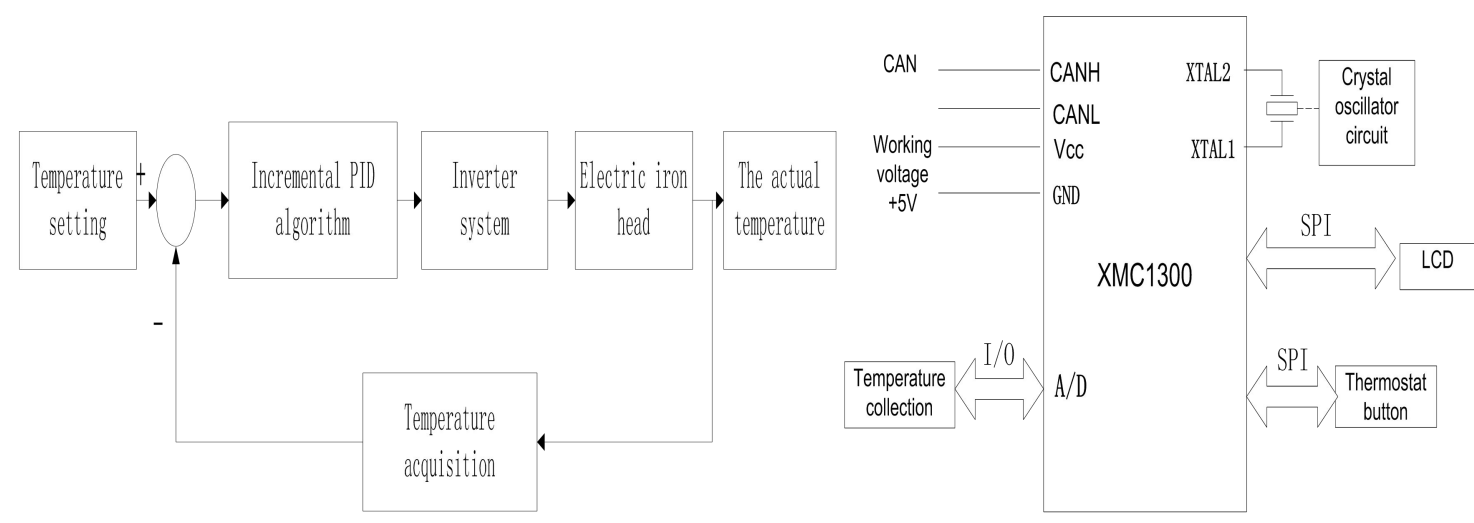

Fig. 4. Block diagram of temperature control system

Fig. 5. Master chip system structure diagram

\section{Master chip selection}

Infineon 32-bit digital signal processor xmc1300 is applied as the main control chip, which uses SPI bus as data real-time transmission. Therefore, the signal is collected by optocoupler for isolation 
of strong and weak electricity, and then sent to the DSP for signal processing, AD conversion and PI algorithm to get adjustable constant temperature control. At the same time, LCD can display the temperature of the iron. The main system peripheral structure of the chip is shown in Figure 5:

\section{PID control algorithm}

The strategy of integral separation PID control is adopted. The basic idea of improved PID is as follow: When the deviation of controlled variable and set point is large, integral action is cancelled to; on the other side, integral action is used to eliminate static error and improve the control precision. Follow the steps outlined below:

Integration separation control arithmetic can be expressed as:

$$
\begin{gathered}
u(k)=K_{p} e(k)+\beta k_{i} \sum_{j=0}^{k} e(j) T+k_{d}(e(k)-e(k-1) / T \\
\beta= \begin{cases}1 & |\operatorname{error}(k)| \leq \varepsilon \\
0 & |\operatorname{error}(k)|>\varepsilon\end{cases}
\end{gathered}
$$

$T$ is sample time ; $\beta$ is coefficient of integral; $\varepsilon$ is threshold value.

\section{Results and conclusion}

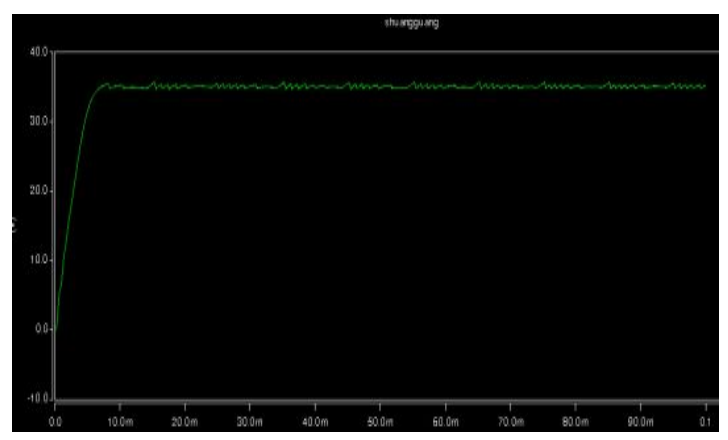

Fig. 6. The power output of the simulation results

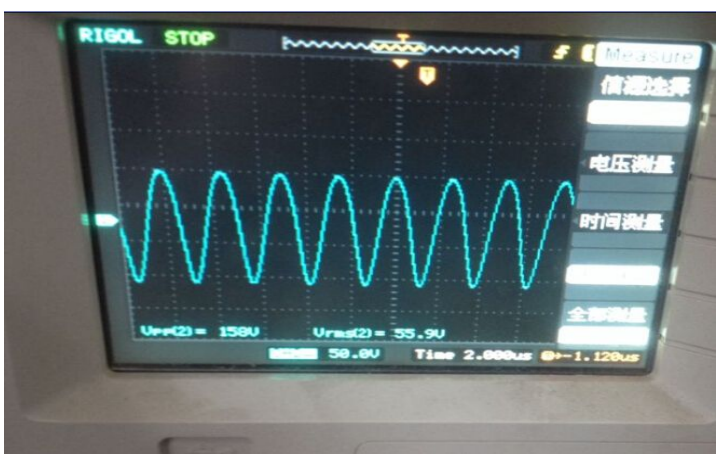

Fig. 7. Voltage inverter diagram

Experiments show that the requirements of the electric iron power feedback system and inverter circuits meet all performance indexes of the system we design. The system has good dynamic response and load adjustment rate is high and stable output voltage and small ripple. This system can provide the electric iron is a good heating, and the temperature can be adjusted to maintain constant temperature, fast heating speed (less than $7 \mathrm{~S}$ heating), and at $200^{\circ} \mathrm{C}-500^{\circ} \mathrm{C}$ degrees adjustable.

\section{References}

[1] PRESSMAN A I, BILLINGS K, MOREY T.Design of switching power supply[M].Wang Zhiqiang,Xiao Wenxun, Yu Long,et al..3.Beijing: Electronic Industry Press.2010:100-142.

[2] $\mathrm{Hu}$ Rongqiang,Huang Qingyi,Wang Chuangrui.Current type pulse width modulator UC3842 peripheral circuit improved[J].electrical applications, 2005,24(7):77280

[3] Fu Qing.Parallel resonant frequency power inverter circuit phase angle automatic application of [J].power electronic technology,2009,43(9):35-37.

[4] KEITH B. switching power supply manual [M].2 version.Zhang Zhansong, Wang Renhuang, Xie Liping,trans.Beijing:People's Posts and Telecommunications Press,2006

[5] Wang Chuangrui.The flyback switching power supply feedback loop[J]. communication power technology,2005,22(2):42-44.

[6] Tao Yonghua. The new PID control and application[J].industrial instrumentation and automation devices.1998(1):57-62. 